\title{
ADICCIÓN A INTERNET: DESARROLLO Y VALIDACIÓN DE UN INSTRUMENTO EN ESCOLARES ADOLESCENTES DE LIMA, PERÚ
}

\author{
Nelly Lam-Figueroa ${ }^{1, a}$, Hans Contreras-Pulache ${ }^{2, b}$, Elizabeth Mori-Quispe ${ }^{2, b}$, Martín Nizama-Valladolid ${ }^{3, d}$, \\ César Gutiérrez ${ }^{4, e}$, Williams Hinostroza-Camposano, ${ }^{2, b}$, Erasmo Torrejón Reyes ${ }^{2, f}$, \\ Richard Hinostroza-Camposano ${ }^{2, b}$, Elizabeth Coaquira-Condori ${ }^{2, b}$, \\ Willy David Hinostroza-Camposano ${ }^{2, c}$
}

\begin{abstract}
RESUMEN
Objetivos. Desarrollar y validar un instrumento que permita evaluar el fenómeno de la adicción a Internet (Al) en adolescentes de Lima Metropolitana. Materiales y métodos. Se realizó un estudio observacional y analítico, donde incluimos una muestra de 248 adolescentes escolares del primer al quinto año de secundaria. Para evaluar la Al construimos el cuestionario 'Escala de la Adicción a Internet de Lima' (EAIL), la cual evalúa características sintomatológicas y disfuncionales. Los ítems resultantes fueron sometidos a un juicio de expertos, determinándose finalmente una escala de once ítems. Resultados. La edad media fue de 14 años. El análisis psicométrico del instrumento presentó un coeficiente Alfa de Cronbach de 0,84 , con valores de correlación ítem-total de 0,45 hasta 0,59. El análisis dimensional mostró una estructura de dos dimensiones que explicaba el 50,7 \% de la varianza total. El análisis bidimensional mostró asociación significativa $(p<0,001)$ entre la Dimensión I (Sintomatología de Al) y el tiempo de uso semanal, sexo masculino, antecedente de problemas de indisciplina y plan de futuro. La Dimensión II (Disfuncionalidad por Al) se asoció significativamente $(p<0,001)$ al antecedente de problemas de indisciplina, plan de futuro y faltar sin motivo justificable a la escuela. Conclusiones. La EAIL presentó una buena consistencia interna, con correlaciones moderadas y significativas entre sus ítems. Los hallazgos evidencian que la adicción cumple un papel dinámico que pone en manifiesto una alteración que involucra en su génesis patrones de familia y entornos sociales inadecuados.
\end{abstract}

Palabras clave: Estudios de validación; Internet; Adicción; Adolescente; Cuestionarios (fuente: DeCS BIREME).

\section{INTERNET ADDICTION: DEVELOPMENT AND VALIDATION OF AN INSTRUMENT IN ADOLESCENT SCHOLARS IN LIMA, PERU}

\begin{abstract}
SUMMARY
Objectives. To develop and validate an instrument to assess Internet Addiction (IA) phenomenon in adolescents of Metropolitan Lima. Materials and methods. We performed an observational analytical study, including a sample of 248 high school adolescent students. In order to evaluate the IA, we constructed the questionnaire: "Scale for Internet Addiction of Lima" (SIAL), which assesses symptoms and dysfunctional characteristics. The resulting items were submitted to experts' judgment, finally obtaining a 11 -item scale. Results. The mean age was 14 years old. The psychometric analysis of the instrument showed a Cronbach' Alpha Coefficient of 0.84 , with values of item-total correlation ranging from 0.45 to 0.59. The dimensional analysis yielded a two-dimensional structure that explained up to $50.7 \%$ of the total variance. The bi-dimensional data analysis revealed a significant association $(p<0,001)$ between Dimension I (symptoms of IA) and the weekly time spent on the Internet, male sex, past history of bad behavior in school and plans for the future. Dimension II (dysfunction due to IA) had a significant association to past history of bad behavior, plans for the future $(p<0,001)$ and missing school without valid reasons. Conclusions. The SIAL showed a good internal consistency, with moderate and significant inter-item correlations. The findings show that addiction has a dynamic role, which evidences a problem generated in family patterns and inadequate social networks.
\end{abstract}

Key words: Validation study; Internet; Addiction; Adolescent; Questionnaires (source: MesH NLM).

\footnotetext{
Instituto de Investigaciones Clínicas. Universidad Nacional Mayor de San Marcos. Lima, Perú.

2 Centro de Investigación ANASTOMOSIS. Universidad Nacional Mayor de San Marcos. Lima, Perú.

3 Instituto Nacional de Salud Mental "Honorio Delgado Hideyo Noguchi”. Lima, Perú.

4 Instituto de Medicina Tropical "Daniel A. Carrión”. Universidad Nacional Mayor de San Marcos. Lima, Perú.

a Médico, Ginecobstetra, Magíster en Medicina; ${ }^{\mathrm{b}}$ Médico Cirujano; ${ }^{\mathrm{C}}$ Bachiller en Medicina Humana; ${ }^{\mathrm{d}}$ Médico, Psiquiatra, Doctor en Medicina; ${ }^{\mathrm{e}}$ Médico, Magíster en Epidemiología; ${ }^{\mathrm{f}}$ Médico, Cardiólogo y Cardiocirujano, Doctor en Medicina

* Este trabajo fue presentado como resumen en el VI Concurso Nacional de Investigación en Salud de Adolecentes y Jóvenes "María Miranda Lozano", 2010, organizado por la Sociedad Peruana de Adolescencia y Juventud.
}

Recibido: 10-03-11 Aprobado: 24-08-11 


\section{INTRODUCCIÓN}

Uno de los acontecimientos de mayor trascendencia en los últimos años, ha sido el progresivo impacto del uso de nuevas tecnologías en los estilos de vida y en el desarrollo de las relaciones de socialización (1). Así, al mismo tiempo que Internet se ha posicionado como una herramienta ventajosa, aparecen en distintas localidades informes sobre las consecuencias del abuso y descontrol en su utilización, conocidos son ya los términos "ciberadicción”, "netadicción”, "desorden de adicción a Internet", "uso compulsivo de Internet", "uso patológico de Internet" o simplemente "adicción a Internet" (AI) ${ }^{(2-5)}$. Sin embargo, luego de más de una década de investigación académica, el concepto de Al aún no ha sido del todo desarrollado, y bien podríamos decir, conjuntamente con una revisión reciente ${ }^{(6)}$, que una aproximación concreta y holística al fenómeno de la Al se encuentra todavía en desarrollo.

Introducido por Goldberg en $1995{ }^{(7)}$ y popularizado luego por Young en $1998{ }^{(4)}$, el concepto de Al ha pasado por varias modificaciones según el enfoque recibido ${ }^{(6)}$. Prueba de ello son los criterios diagnósticos de Young (2); modificados luego por Shapira ${ }^{(8)}$; las aproximaciones de Beard ${ }^{(9)}$; los intentos de correlación con conceptos como autoestima, timidez y depresión efectuados por Caplan (10); los aportes de Davis y su modelo cognitivo conductual, aplicado sobre todo para evaluar a personas aptas para trabajos de alta exposición (11); Charlton y la distinción del High engagement (alto involucramiento) antes que la adicción (12,13); las experiencias cualitativas recogidas por Douglas y colaboradores ${ }^{(6)}$ y su modelo teórico del fenómeno y, finalmente, los modelos experimentales provenientes de Asia (Japón, Korea y Taiwan) entre los cuales resaltamos los aportes (en especial lo referido a los criterios diagnósticos) de Ko y colaboradores ${ }^{(14)}$.

En un intento de comprensión global, y a modo de construcción teórica propia, podríamos decir que la Al se caracteriza por una incapacidad de la persona para ejercer control sobre el uso y dependencia a Internet, lo que lo lleva a estados de fatiga, incomodidad y falla en su devenir cotidiano ${ }^{(15)}$. Al mismo tiempo, una persona como tal presentará estados de alta tensión o angustia antes del uso de Internet, los cuales cesarán una vez lograda la conexión al mundo virtual ${ }^{(2,6,8,16,17)}$.

Internet se ha posicionado como una herramienta útil, global e inevitable; y es debido a estas características que toda persona, de uno u otro modo, llega a desarrollar una interrelación con el mundo virtual, lo que conlleva el establecimiento de estados de dependencia (no necesariamente patológicos) a Internet. Con relación a la $\mathrm{Al}$, una persona primero desarrolla un "estado de involucramiento a Internet", es decir, un estado de uso de los servicios que se brindan a través de Internet; y que según las necesidades en las que se vea envuelta la persona será menor o mayor, o lo que Charlton define como bajo o alto involucramiento ${ }^{(13)}$. Como resultado de esta dinámica de/en la persona (en la que entran a tallar sus condiciones económicas y de desarrollo moral, sus actitudes como medios para plantearse retos en la vida, objetivos a lograse en el corto y largo plazo) se podrían desarrollar conductas mal adaptativas (es decir, de uso patológico de Internet) con repercusiones directas en la vida cotidiana de la persona; a todo ello, en conjunto, llamamos 'Adicción a Internet', cuyo problema no es posible negar ${ }^{(5,6,16-20)}$.

Esta adicción podrá definirse como la imposibilidad para controlar el involucramiento y tendrá "características sintomatológicas" (significativamente distinguibles de los estados de normal involucramiento) y "características disfuncionales" (y es en tanto a estas disfuncionalidades o consecuencias que se evaluará como entidad problemática). Esta segunda dimensión (de las consecuencias) no ha sido muy considerada en las publicaciones provenientes principalmente de Asia y Norteamérica, lo que ha resultado en una comprensión incompleta del panorama de la $\mathrm{Al}{ }^{(13-21)}$, llegándose a considerar como adicto en muchos casos a la persona altamente involucrada. Por ello, no se tienen estadísticas confiables relacionadas con la prevalencia de la Al.

En Perú, según lo revelado por APOYO el $2008{ }^{(22)}$, el grupo adolescente es el que mayor exposición presenta a las tecnológicas digitales y, entre ellas, a Internet. En este contexto es que planteamos la siguiente investigación, con el objetivo de desarrollar (luego de un enfoque cualitativo y cuantitativo) y validar un instrumento que permita evaluar la $\mathrm{Al}$ en adolescentes.

\section{MATERIALES Y MÉTODOS}

\section{POBLACIÓN, CRITERIOS DE SELECCIÓN Y MUESTREO}

Se realizó un estudio observacional y analítico, involucrando a una población de adolescentes escolarizados pertenecientes a instituciones educativas públicas de Lima Metropolitana, dependientes de la Unidad de Gestión Educativa Local N. 02 (UGEL 02). Se seleccionó de modo aleatorio una población que incluyó colegios de varones, de mujeres y mixtos. Los centros educativos incluidos en el presente estudio fueron el colegio de varones Leoncio Prado N. ${ }^{\circ} 3014$, el colegio de mujeres Lucie Rynning de Antúnez de Mayolo, y los colegios mixtos 
Tabla 1. Escala de Adicción a Internet de Lima (EAIL).

\begin{tabular}{|c|c|c|c|c|c|}
\hline & ÍTEM & $\begin{array}{l}\text { Muy rara } \\
\text { vez }\end{array}$ & $\begin{array}{l}\text { Rara } \\
\text { vez }\end{array}$ & $\begin{array}{c}\text { A } \\
\text { menudo }\end{array}$ & Siempre \\
\hline 1 & $\begin{array}{l}\text { Cuando no estoy conectado, imagino cómo será la próxima vez que estaré co- } \\
\text { nectado a Internet }\end{array}$ & & & & \\
\hline 2 & Me siento preocupado o sueño con conectarme cuando no lo estoy & & & & \\
\hline 3 & Luego de haberme desconectado, siento que aún quiero estar en Internet & & & & \\
\hline 4 & $\begin{array}{l}\text { Me siento deprimido, malhumorado o nervioso cuando no estoy conectado y se } \\
\text { me pasa cuando vuelvo a conectarme }\end{array}$ & & & & \\
\hline 5 & Dedico más tiempo a Internet del que pretendía & & & & \\
\hline 6 & Digo a menudo "sólo unos minutitos más" cuando estoy conectado & & & & \\
\hline 7 & He intentado estar menos tiempo conectado pero no he podido & & & & \\
\hline 8 & Cuando siento el impulso de conectarme a Internet no lo puedo controlar & & & & \\
\hline 9 & He llegado a perder clases por estar en Internet & & & & \\
\hline 10 & He tenido problemas en el colegio por estar en Internet & & & & \\
\hline 11 & Descuido las tareas domésticas por pasar más tiempo conectado & & & & \\
\hline
\end{tabular}

José Granda y Ramón Castilla N. ${ }^{\circ} 3043$. Estos cuatro centros educativos son los que presentaron mayor volumen estudiantil según los informes que se obtuvieron de los registros de la Unidad de Estadística de la Calidad Educativa de la UGEL 02 del Ministerio de Educación. Los 4430 estudiantes de estos cuatro colegios, estaban distribuidos en 148 secciones. Se estimó en EPIDAT versión 3.1 un tamaño de muestra para una proporción poblacional, con una frecuencia esperada de $10 \%$ de adicción a Internet entre escolares, un nivel de confianza del $95 \%$ y una precisión del $4 \%$, obteniendo un tamaño de muestra de 207 escolares. Con el mismo programa, aplicando el módulo de selección de muestras por conglomerados, se consideró como un conglomerado a cada sección de las 148 secciones contenidas en los cuatro colegios y se procedió a una selección por conglomerados monoetápico. Puesto que la selección era aleatoria, se generaron hasta 15 muestras, de tal manera que se llegara a obtener una en la cual se incluyeran secciones de los cinco años de educación secundaria, tanto en turno mañana como tarde. La muestra final obtenida fue de 12 conglomerados, que incluían 248 escolares. Se evaluaron las siguientes variables: edad, sexo, año de estudios, jornada de estudios, modo de acceso a Internet, tiempo promedio por semana, antecedente de haber repetido un año escolar, antecedente de problemas de disciplina, falta injustificada al colegio, plan de futuro, motivos de usos de Internet y Al. La aplicación del instrumento se realizó durante el mes de septiembre de 2009.

\section{DESARROLLO Y VALIDACIÓN DE LA EAIL}

ParaevaluarlaAlseconstruyóelcuestionarioquellamamos 'Escala de la Adicción a Internet de Lima' (EAIL) según un modelo teórico propio (explicado en la introducción) basado en las publicaciones previas ${ }^{(6,8,9,14,23,24)}$. Así, se construyeron ítems que evaluaran las características sintomatológicas: saliencia (estar preocupado por la utilización de Internet), tolerancia, abstinencia, falla en el control y recaída; así como también características disfuncionales: problemas académicos, familiares e interpersonales. Para ello, se revisaron los cuestionarios de autoinforme más significativos en relación a la $\mathrm{Al}$ : Internet Addiction Test (4), Online CognitionScale (11), Chinese Internet Addiction Inventory ${ }^{(25)}$, Generalized Problematic Internet Use Scale ${ }^{(10)}$, Pathological Internet Use Scale ${ }^{(26)}$. Los ítems resultantes fueron sometidos a un juicio de tres psiquiatras especializados en el campo de las adicciones, cuya dirección estuvo a cargo de MNV, determinándose finalmente una escala de once ítems: ocho evalúan las características sintomatológicas y tres evalúan las características disfuncionales. Cada ítem cuenta con respuestas de opción múltiple tipo Likert de cuatro opciones: Muy rara vez (1), Rara vez (2), A menudo (3), Siempre (4). La EAIL que desarrollamos se muestra en la Tabla 1.

De forma paralela, se procedió a entrevistar a un grupo de pacientes internados en el Instituto Nacional de Salud Mental "Honorio Delgado Hideyo Noguchi" con el diagnóstico de Al según los criterios del DSM-IV para desórdenes en el control de impulsos o juego patológico. Las entrevistas estuvieron a cargo de HCP y MNV. Estas entrevistas tuvieron como objetivo generar un perfil de características de la persona adicta a partir del cual se pudiera elaborar "ítems de corroboración" para comprobar luego la validez de la EAIL. Así, se generó la siguiente hipótesis, aquellos que refirieran respuestas de mayor frecuencia a los "ítems de corroboración" deberían obtener mayores puntajes, significativamente hablando, en la EAIL. Los "ítems de corroboración" fueron los ocho siguientes: (1) Temo que la vida sin Internet sea aburrida vacía y sin alegría, (2) Estar en Internet puede reducir el estrés de la vida real y del día a día, (3) Suelo acortar mi tiempo de sueño para estar más tiempo en Internet, (4) 
Internet es el mejor sitio para pasar el rato, (5) Respondo de mala manera, grito o me enfado si me molestan cuando estoy conectado, (6) Internet es lo más importante en mi vida, (7) Recibo quejas de otras personas por la cantidad de tiempo que paso en Internet, (8) Intento ocultarle a los demás el tiempo que realmente estoy conectado. El proyecto y la EAIL fueron aprobados por el Comité de Ética del Instituto Nacional de Salud Mental "Honorio Delgado Hideyo Noguchi” de Lima, Perú.

\section{ANÁLISIS ESTADÍSTICO}

El análisis se hizo centrado en evaluación de la EAIL. Se calcularon medidas de consistencia interna, mediante el coeficiente Alfa de Cronbach, así como de correlación, mediante el coeficiente de correlación de Pearson. Para evaluar las dimensiones subyacentes a la EAIL se realizó un análisis factorial, previo a lo cual se determinó la existencia de relaciones significativas entre las variables determinándose el Test de Esfericidad de Bartlett y la Medida de Adecuación de la Muestra KMO. Para las variables cualitativas se analizaron las frecuencias y proporciones, para las variables cuantitativas se determinaron valores de tendencia central y medidas de dispersión. Para el análisis diferencial de medias se utilizó chi cuadrado y ANOVA con pruebas post-hoc. Se consideró un intervalo de confianza al $95 \%$ y a todo valor de $\mathrm{p}<0,05$ como estadísticamente significativo.

\section{RESULTADOS}

La muestra estudiada de 248 estudiantes, se caracteriza en la Tabla 2 en relación a variables demográficas escolares y de acceso a Internet.

Tabla 2. Características demográficas y acceso a Internet en adolescentes escolarizados de Lima-Perú, 2009.

\begin{tabular}{lr}
\hline \multicolumn{1}{c}{ Variable } & $\mathbf{n}(\%)$ \\
\hline Sexo & $133(53,6)$ \\
Masculino & $115(36,4)$ \\
Femenino & \\
Año de estudio & $53(21,4)$ \\
Primer año & $105(42,3)$ \\
Segundo año & $29(11,7)$ \\
Tercer año & $45(18,1)$ \\
Cuarto año & $16(6)$ \\
Quinto año & $168(67,7)$ \\
Jornada de estudio & $80(32,3)$ \\
Mañana & \\
Tarde & $81(32,7)$ \\
Acceso a Internet & $167(67,3)$ \\
En casa & \\
Cabinas & $159(64,1)$ \\
Tiempo de uso de Internet & $51(20,6)$ \\
Menos de 5 horas semanales & $38(15,3)$ \\
Entre 6 y 10 horas semanales & \\
Más de 10 horas semanales &
\end{tabular}

Tabla 3. Valoración de la importancia de los motivos por los cuales se usa Internet en adolescentes escolarizados de Lima-Perú, 2009.

\begin{tabular}{lccc}
\hline \multirow{2}{*}{$\begin{array}{l}\text { Internet es importante } \\
\text { para: }\end{array}$} & $\begin{array}{c}\text { Nada } \\
\text { importante }\end{array}$ & $\begin{array}{c}\text { Más o } \\
\text { menos } \\
\text { importante }\end{array}$ & $\begin{array}{c}\text { Muy } \\
\text { importante }\end{array}$ \\
\cline { 2 - 4 } & $\mathbf{n}(\%)$ & $\mathbf{n}(\%)$ & $\mathbf{n}(\%)$ \\
\hline $\begin{array}{l}\text { Conocer personas } \\
\begin{array}{l}\text { Comunicarse con } \\
\text { conocidos }\end{array}\end{array}$ & $46(18,5)$ & $129(52)$ & $73(29,5)$ \\
\hline $\begin{array}{l}\text { Tener un espacio } \\
\text { personal }\end{array}$ & $23(9,3)$ & $113(45,5)$ & $112(45,2)$ \\
$\begin{array}{l}\text { Buscar información } \\
\text { para adultos }\end{array}$ & $173(69,8)$ & $61(24,6)$ & $14(5,6)$ \\
\hline \begin{tabular}{l} 
Jugar en red \\
\hline
\end{tabular} & $44(17,7)$ & $122(49,2)$ & $82(33,1)$ \\
\hline
\end{tabular}

En el plano educativo, el 36,3\% de los estudiantes refirió haber desaprobado al menos un año escolar. Cuando se preguntó por el antecedente de problemas disciplinarios, el 41,9 \% refirió nunca tener problemas, 52,4\% refirió tener problemas esporádicamente y 5,6 \% refirió tener problemas con frecuencia. El antecedente de haber faltado a la escuela sin motivo justificable se presentó en el 17,7\% de los adolescentes estudiados. Con relación a sus planes a futuro, el 4,4 \% refirió que una vez terminado el colegio se pondrían a trabajar, el $24,6 \%$ mostró interés por seguir una carrera técnica, $53,2 \%$ por continuar estudios universitarios, 9,1\% refirió deseos de ingresar a las fuerzas armadas mientras que un $9,7 \%$ refirió no tener en claro el rumbo de su futuro.

Los motivos de usos de Internet fueron: conocer personas, comunicarse con conocidos, tener un espacio personal, buscar información para adultos, según importancia, se muestran en la Tabla 3.

El análisis psicométrico del instrumento planteado por los autores (EAIL) presentó un coeficiente alfa de Cronbach de 0,84 , si retiramos cualquier ítem, este coeficiente bajaría a 0,82 o 0,83 en todos los casos. Mientras, los valores de correlación ítem total variaron desde 0,45 hasta 0,69 , como se muestra en la Tabla 4.

Para definir si procedía un análisis factorial, primero se determinó si las variables evaluadas a través de los ítems de la EAIL presentaban relaciones significativas. El test de esfericidad de Bartlett arrojó un estadístico de 807,26 con un $p<0,0001$. La medida de adecuación muestral KMO fue de 0,851. Dados los resultados anteriores, se procedió entonces a la realización de un análisis factorial a fin de estudiar la estructura subyacente de la EAIL en términos de factores o dimensiones. Las 
Tabla 4. Análisis de consistencia interna mediante coeficiente alfa de Cronbach de la Escala de la Adicción a Internet de Lima (EAIL).

\begin{tabular}{lcc}
\hline \multicolumn{1}{c}{ EAIL } & $\begin{array}{c}\text { Correlación } \\
\text { ítem-total }{ }^{*}\end{array}$ & $\begin{array}{c}\text { Alfa si el } \\
\text { ítem se } \\
\text { elimina }\end{array}$ \\
\hline $\begin{array}{l}\text { Cuando no estoy conectado, } \\
\text { imagino cómo será la próxima vez } \\
\text { que estaré conectado a Internet }\end{array}$ & 0,63 & 0,82 \\
$\begin{array}{l}\text { Me siento preocupado o sueño con } \\
\text { conectarme cuando no lo estoy }\end{array}$ & 0,64 & 0,82 \\
$\begin{array}{l}\text { Luego de haberme desconectado, } \\
\text { siento que aún quiero estar en } \\
\text { Internet }\end{array}$ & 0,61 & 0,83 \\
$\begin{array}{l}\text { Me siento deprimido, malhumorado } \\
\text { o nervioso cuando no estoy } \\
\text { conectado y se me pasa cuando } \\
\text { vuelvo a conectarme }\end{array}$ & 0,66 & 0,82 \\
$\begin{array}{l}\text { Dedico más tiempo a Internet del } \\
\text { que pretendía }\end{array}$ & 0,57 & 0,83 \\
$\begin{array}{l}\text { Digo a menudo "solo unos minutitos } \\
\text { más" cuando estoy conectado }\end{array}$ & 0,61 & 0,83 \\
$\begin{array}{l}\text { He intentado estar menos tiempo } \\
\text { conectado pero no he podido }\end{array}$ & 0,59 & 0,83 \\
$\begin{array}{l}\text { Cuando siento el impulso } \\
\text { conectarme a Internet no lo puedo } \\
\text { controlar }\end{array}$ & 0,69 & 0,82 \\
$\begin{array}{l}\text { He llegado a perder clases por } \\
\text { estar en Internet } \\
\text { He tenido problemas en el colegio } \\
\text { por estar en Internet }\end{array}$ & 0,57 & 0,83 \\
$\begin{array}{l}\text { Descuido las tareas domésticas } \\
\text { por pasar más tiempo conectado }\end{array}$ & 0,66 & 0,82 \\
\hline * En todos casos p & & \\
\hline
\end{tabular}

* En todos los casos $p<0,001$

dos dimensiones encontradas explicaron el 50,7 \% de la varianza total. La Tabla 5 resume los valores del análisis factorial exploratorio a la que se aplicó una rotación varimax con el objeto de aumentar la dispersión de las ponderaciones factoriales y facilitar la interpretación de los factores.

El análisis correlacional reveló que los ítems de la Dimensión I (Características Sintomatológicas de la $\mathrm{Al}$ ) presentaron correlaciones (según coeficiente de Pearson) desde 0,22 a 0,42 ( $p<0,001)$, mientras que las correlaciones de los ítems de la Dimensión II (Características Disfuncionales de la Al) oscilaron entre 0,51 y 0,59 $(p<0,001)$.

El análisis bidimensional mostró una asociación significativa $(p<0,001)$ entre la Dimensión I y el tiempo de uso semanal (asociación directa), sexo (más compromiso en varones que en mujeres), antecedente de problemas de indisciplina (asociación directa) y plan de futuro (más altos índices en aquellos que deseaban ingresar a las fuerzas armadas y en los que no tenían un rumbo claro de futuro). La Dimensión II se asoció significativamente $(p<0,001)$ al antecedente de problemas de indisciplina (asociación directa), plan de futuro (del mismo modo que la Dimensión I), falta sin motivo justificable a la escuela (mayores índices en aquellos que refirieron haberse "tirado la pera").

Existió una correlación significativa y directa entre los valores de Dimensión I y Dimensión II en relación al grado de importancia que la persona otorga a los motivos de uso de Internet, como se ve en la Tabla 6.

En relación a la hipótesis planteada para evaluar la consistencia de la EAIL, a manera de confirmación del constructo teórico, se presenta el resultado del análisis correlacional entre la EAIL y los "ítems de corroboración" en la Tabla 7.

Tabla 5. Análisis factorial exploratorio de la Escala de la Adicción a Internet de Lima (EAIL).

\begin{tabular}{|c|c|c|}
\hline Escala de la Adicción a Internet de Lima & $\begin{array}{c}\text { Dimensión I } \\
\text { características } \\
\text { sintomatológicas de Al }\end{array}$ & $\begin{array}{c}\text { Dimensión II } \\
\text { características } \\
\text { disfuncionales por Al }\end{array}$ \\
\hline $\begin{array}{l}\text { Cuando no estoy conectado, imagino cómo será la próxima vez que } \\
\text { estaré conectado a Internet }\end{array}$ & 0,59 & 0,14 \\
\hline Me siento preocupado o sueño con conectarme cuando no lo estoy & 0,63 & 0,22 \\
\hline $\begin{array}{l}\text { Luego de haberme desconectado, siento que aún quiero estar en } \\
\text { Internet }\end{array}$ & 0,50 & 0,42 \\
\hline $\begin{array}{l}\text { Me siento deprimido, mal humorado o nervioso cuando no estoy } \\
\text { conectado y se me pasa cuando vuelvo a conectarme }\end{array}$ & 0,66 & 0,11 \\
\hline Dedico más tiempo a Internet del que pretendía & 0,66 & 0,08 \\
\hline Digo a menudo "solo unos minutitos más" cuando estoy conectado & 0,48 & 0,44 \\
\hline He intentado estar menos tiempo conectado pero no he podido & 0,66 & 0,27 \\
\hline Cuando siento el impulso conectarme a Internet no lo puedo controlar & 0,65 & 0,14 \\
\hline He llegado a perder clases por estar en Internet & 0,31 & 0,69 \\
\hline He tenido problemas en el colegio por estar en Internet & 0,12 & 0,86 \\
\hline Descuido las tareas domésticas por pasar más tiempo conectado & 0,08 & 0,83 \\
\hline
\end{tabular}


Tabla 6. Análisis correlacional entre los motivos de uso de Internet, según importancia, y las dos dimensiones de la Escala de la Adicción a Internet de Lima (EAIL).

\begin{tabular}{|c|c|c|c|c|c|}
\hline $\begin{array}{l}\text { Internet } \\
\text { importante }\end{array}$ & $\begin{array}{l}\text { es } \\
\text { oara: }\end{array}$ & Dimensión & $\begin{array}{c}\text { valor } \\
p\end{array}$ & $\begin{array}{c}\text { Dimensión } \\
\text { II }\end{array}$ & $\begin{array}{c}\text { valor } \\
p\end{array}$ \\
\hline & $\mathrm{NI}^{*}$ & 13,5 & & 3,9 & \\
\hline Conocer & $\mathrm{MMI}^{\dagger}$ & 15,5 & $<0,001$ & 4,3 & $<0,001$ \\
\hline & $M l^{\ddagger}$ & 16,1 & & 4,5 & \\
\hline & $\mathrm{NI}$ & 14,3 & & 4,5 & \\
\hline Comunicarse & MMI & 15,3 & 0,605 & 4,3 & 0,916 \\
\hline & MI & 15,5 & & 4,3 & \\
\hline Tener un & $\mathrm{NI}$ & 13,5 & & 4 & \\
\hline espacio & MMI & 14,6 & $<0,001$ & 4,1 & $<0,001$ \\
\hline personal & MI & 17,3 & & 4,7 & \\
\hline Buscar & $\mathrm{NI}$ & 14,4 & & 4,2 & \\
\hline información & MMI & 17,2 & $<0,001$ & 4,6 & 0,143 \\
\hline para adultos & MI & 17,6 & & 5,1 & \\
\hline & $\mathrm{NI}$ & 14,2 & & 3,9 & \\
\hline Jugar en red & MMI & 14,9 & $<0,05$ & 4,2 & $<0,05$ \\
\hline & MI & 16,4 & & 4,7 & \\
\hline
\end{tabular}

* NI: Nada Importante. † MMI: Más o menos importante. ₹ MI: Muy importante.

\section{DISCUSIÓN}

Comomuestranlos resultados enlapresente investigación, la EAIL presenta una alta consistencia interna, con correlaciones moderadas y significativas entre sus ítems. Las pruebas psicométricas utilizadas para justificar el análisis factorial fueron el test de esfericidad de Bartlett (que evalúa si la matriz de correlaciones es una matriz de identidad) y la medida de adecuación muestral KMO (que compara los coeficientes de correlación observados con los coeficientes de correlación parcial). En nuestro estudio, el test de esfericidad de Barlett tuvo un valor adecuado además de significativo, y la medida de adecuación muestral KMO tuvo un valor de 0,851, siendo recomendado que sea mayor de 0,5. En base a estos dos resultados favorables, se procedió al análisis factorial de la EAIL, el cual confirmó el supuesto teórico según el cual se basó el diseño de la escala (dos dimensiones), y que en resumidas cuentas plantea que el fenómeno de la Al involucra tanto una dimensión sintomatológica como una dimensión de disfuncionalidades en la vida de la persona, esta concepción ha sido ya reiterada en estudios previos ${ }^{(4-6,8,14)}$.

Por otro lado, la hipótesis planteada, y que sirvió para la corroboración de la validez de la EAIL, resultó confirmada en el análisis correlacional encontrándose una asociación significativa y directa entre los "ítems de corroboración" y la EAIL, tanto en su Dimensión I (Sintomatología de Al) como en su Dimensión ||
Tabla 7. Análisis correlacional entre la Escala de la Adicción a Internet de Lima (EAIL) y los "ítems de corroboración".

\begin{tabular}{|c|c|c|c|c|c|}
\hline \multicolumn{2}{|c|}{ Criterios clínicos } & \multirow{2}{*}{$\begin{array}{c}\text { Dimensión } \\
12,9\end{array}$} & \multirow[t]{2}{*}{ valor $p$} & \multirow{2}{*}{$\begin{array}{c}\text { Dimensión } \\
\text { II } \\
3,9\end{array}$} & \multirow[t]{2}{*}{$\begin{array}{c}\text { valor } \\
p\end{array}$} \\
\hline \multirow{4}{*}{$\begin{array}{l}\text { Temo que la vida } \\
\text { sin Internet sea } \\
\text { aburrida, vacía y } \\
\text { sin alegría }\end{array}$} & $\mathrm{MRV}^{*}$ & & & & \\
\hline & $\mathrm{RV}^{\dagger}$ & 15,6 & $<0,001$ & 4,4 & \multirow{3}{*}{$<0,05$} \\
\hline & $\mathrm{AM}^{\ddagger}$ & 18,2 & & 4,6 & \\
\hline & $\mathrm{MM}^{\S}$ & 19,8 & & 5,2 & \\
\hline \multirow{4}{*}{$\begin{array}{l}\text { Estar en Internet } \\
\text { puede reducir el } \\
\text { estrés de la vida } \\
\text { real y del día a día }\end{array}$} & MRV & 12,9 & \multirow{4}{*}{$<0,001$} & 3,7 & \multirow{4}{*}{$<0,001$} \\
\hline & RV & 14,9 & & 4,4 & \\
\hline & AM & 18,1 & & 4,9 & \\
\hline & MM & 19,2 & & 4,9 & \\
\hline \multirow{4}{*}{$\begin{array}{l}\text { Suelo acortar mi } \\
\text { tiempo de sueño } \\
\text { para estar más } \\
\text { tiempo en Internet }\end{array}$} & MRV & 13,4 & \multirow{4}{*}{$<0,001$} & 3,7 & \multirow{4}{*}{$<0,001$} \\
\hline & RV & 16,8 & & 4,5 & \\
\hline & $\mathrm{AM}$ & 18,4 & & 5,3 & \\
\hline & MM & 20,6 & & 6,6 & \\
\hline \multirow{4}{*}{$\begin{array}{l}\text { Internet es el mejor } \\
\text { sitio para "pasar } \\
\text { el rato" }\end{array}$} & MRV & 12,3 & \multirow{4}{*}{$<0,001$} & 3,5 & \multirow{4}{*}{$<0,001$} \\
\hline & RV & 14,5 & & 4,5 & \\
\hline & AM & 16,4 & & 4,4 & \\
\hline & MM & 19,6 & & 5,2 & \\
\hline \multirow{4}{*}{$\begin{array}{l}\text { Respondo de mala } \\
\text { manera, grito o me } \\
\text { enfado si me mo- } \\
\text { lestan cuando estoy } \\
\text { conectado }\end{array}$} & MRV & 13,5 & \multirow{4}{*}{$<0,001$} & 3,7 & \multirow{4}{*}{$<0,001$} \\
\hline & RV & 16,6 & & 4,8 & \\
\hline & $\mathrm{AM}$ & 18,5 & & 5,4 & \\
\hline & MM & 21,9 & & 6,2 & \\
\hline \multirow{4}{*}{$\begin{array}{l}\text { Internet es lo más } \\
\text { importante en mi } \\
\text { vida }\end{array}$} & MRV & 13,8 & \multirow{4}{*}{$<0,001$} & 3,8 & \multirow{4}{*}{$<0,001$} \\
\hline & RV & 16,6 & & 4,6 & \\
\hline & AM & 18,8 & & 5,7 & \\
\hline & MM & 22 & & 6,8 & \\
\hline \multirow{4}{*}{$\begin{array}{l}\text { Recibo quejas de } \\
\text { otras personas } \\
\text { por la cantidad de } \\
\text { tiempo que paso en } \\
\text { Internet }\end{array}$} & MRV & 13,6 & \multirow{4}{*}{$<0,001$} & 3,7 & \multirow{4}{*}{$<0,001$} \\
\hline & $\mathrm{RV}$ & 16,5 & & 4,3 & \\
\hline & AM & 17,4 & & 5,5 & \\
\hline & MM & 20,8 & & 6,9 & \\
\hline \multirow{4}{*}{$\begin{array}{l}\text { Intento ocultarle a } \\
\text { los demás el tiempo } \\
\text { que realmente } \\
\text { estoy conectado }\end{array}$} & MRV & 13,3 & \multirow{4}{*}{$<0,001$} & 3,6 & \multirow{4}{*}{$<0,001$} \\
\hline & RV & 17 & & 4,8 & \\
\hline & AM & 19,5 & & 5,4 & \\
\hline & MM & 20,3 & & 7,4 & \\
\hline
\end{tabular}

* MRV: Muy rara vez. ${ }^{\dagger}$ RV: Rara vez. ${ }^{\ddagger}$ AM: A menudo. $\S$ MM: Muy a menudo.

(Disfuncionalidad por Al); que los adolescentes que refieren un futuro incierto, así como los que refieren antecedentes de faltas injustificadas a la escuela y problemas de disciplina, presenten mayores índices en la EAIL evidenciaría que el problema de la adicción implica una compleja relación social de la persona, en el que la adicción no cumple un papel independiente o aislado sino que pone en manifiesto una alteración que involucraría en su génesis patrones de familia y entorno social inadecuados, esto debido a que las adicciones son en realidad fenómenos familiares y sociales más que eventos personales o individuales ${ }^{(27)}$.

La adolescencia, con sus características sociobiológicas propias, es un periodo de transición y una etapa en la 
vida de la persona donde acontecen grandes cambios en el sistema de la personalidad (28). Es en este periodo que el sujeto, debido al asentamiento de su naturaleza neurobiológica (el establecimiento del neocórtex conativo) y social (su integración en las relaciones de producción económica), se encuentra particularmente predispuesto a las nuevas tendencias tecnológicas $y$, con ello, a una serie de problemas, en algunos casos con consecuencias desastrosas en la vida de la persona y, finalmente, en el equilibrio de su entorno familiar (15). En el campo de la $\mathrm{Al}$, aún resta mucho por investigar, sobre todo en nuestro país donde no existen datos poblacionales en torno al tema. Creemos que el aporte que hacemos es significativo en cuanto otorga la posibilidad de desarrollar nuevos estudios de carácter local, nacional, utilizando el instrumento desarrollado, no solo para corroborar su validez sino para complementar el conocimiento en torno a la $\mathrm{Al}$, es decir desentrañar su trama patológica: disfunciones personales, disfunciones familiares y disfunciones sociales, son aspectos que se abren para una investigación científica social, cuyo fin último deberá ser la implementación de medidas de salud pública que permitan el control de los fenómenos que, como país pobre subdesarrollado, se constituyen en las razones de nuestra pobreza.

Usualmente, para evaluar el problema de la Al las investigaciones en Latinoamérica han adaptado cuestionarios extranjeros ${ }^{(32)}$, los cuales han sido diseñados en una realidad distinta. La nuestra motiva el desarrollo de instrumentos propios y que estos sean válidos, y se adecuen no solo para el diagnóstico de una enfermedad sino para la intervención sobre ella. Nuestra ayuda a la consecución de dicha meta ha sido la de plantear un instrumento, válido y confiable $(14,15,17,20,30)$. La EAIL no es la traducción de un cuestionario sino la elaboración de uno a partir de nuestra propia comprensión del fenómeno de las adicciones, es decir a partir de nuestra propia concepción del problema y, por tanto, de nuestras motivaciones y actitudes hacia dicho problema.

Estudios extranjeros, esencialmente americanos, han enfocado el problema de la Al desde una perspectiva de mercado o del individuo. Así, el modelo cognitivo conductual desarrolló un instrumento que sirviera a las empresas a identificar a personas en riesgo de desarrollar una Al y que, por tanto, no sean aptas para el desempeño en cierto tipo de labores (11). Nos preguntamos entonces ¿es válido utilizar dicha metodología en nuestra condición de país emergente? Por otro lado, el resto de modelos teóricos se han planteado para entender la problemática de la $\mathrm{Al}$, como un problema de adicción perteneciente al individuo, $y$ es por tanto él quien presenta asociaciones con baja autoestima, sintomatología depresiva, soledad, aislamiento, etc ${ }^{(7,12,23,25,31)}$. ¿Tendrá algo que ver en esta concepción del modelo la estructuración, por ejemplo, que se tiene de la familia en los países de los Estados Unidos y Asia? En este campo, como en toda la medicina, es ineludible el fondo telúrico de nuestras concepciones sociales. En el caso de la Al en particular y de la adicción en general, debe incluir dejar de pensar en la persona de modo aislado y pensar en la familia, para destinar medidas de tipo familiar más que individual ${ }^{(27,28)}$.

En cuanto a los limitantes del presente estudio, podemos discutir en torno a si se debe tomar como suficiente la muestra elegida y si los estudiantes de las cuatro instituciones de educación pública, representativos de la UGEL 02, son válidamente representativas de las instituciones de Lima, sean públicas o privadas $y$, finalmente, de todas las instituciones del Perú o de alguna otra latitud. Esto creemos debe ser evaluado en próximos estudios, en donde la Escala de la Adicción a Internet de Lima debe ser vuelta a poner rigurosamente a prueba, la escala no está limitada para estudiantes de Lima, sino que es desarrollada en esta ciudad y a partir de ella hacia el resto de comunidades. Es papel ahora de los investigadores en este campo el seguir probando la validez de nuestra escala así como también delimitando y aproximándose de manera científica a la problemática de la Al.

En conclusión, la EAIL presentó una buena consistencia interna, con correlaciones moderadas y significativas entre sus ítems. Los hallazgos evidencian que la adicción cumple un papel dinámico que pone en manifiesto una alteración que involucra en su génesis patrones de familia y entornos sociales inadecuados.

\section{Contribuciones de autoría}

NLF, HCP, EMQ y MNV participaron en la concepción y diseño del trabajo, NLF en el análisis e interpretación de datos, en la revisión del informe final y en la obtención del financiamiento. HCP en la recolección de resultados, en el análisis e interpretación de datos, en la redacción del manuscrito y en la obtención de financiamiento. EMQ en la recolección de resultados, en el análisis e interpretación de datos y en la asesoría estadística. MNV en el análisis e interpretación de datos y en el aporte de pacientes o material de estudio. CGV participó en el análisis e interpretación de datos y la revisión crítica del manuscrito. WDHC participó en la recolección de resultados y en la obtención de financiamiento. ETR, RHC, ECC y DHC participaron en la recolección de resultados, en la revisión crítica del manuscrito y en la obtención de financiamiento. Todos los autores aprobaron la versión final del trabajo. 


\section{Fuentes de financiamiento}

Autofinanciado.

\section{Conflictos de interés}

Los autores declaran no tener ningún conflicto de interés.

\section{REFERENCIAS BIBLIOGRÁFICAS}

1. Baruch Y. The autistic society. Information \& Management. 2001;38:129-36.

2. Luengo A. Adicción a Internet: conceptualización y propuesta de intervención. Rev prof esp ter cogn.-conduct. 2004;2:22-52.

3. Young K. Internet addiction: The emergence of a new clinical disorder. Cyberpsychol Behav. 1998;1:237-44.

4. Young K. Internet addiction over the decade: a personal look back. World Psychiatry. 2010;9(2):91.

5. Douglas AC, Mills JE, Niang M, Stepchenkova S, Byun S, Ruffini C, et al. Internet addiction: Meta-synthesis of qualitative research for the decade 1996-2006. Computers in Human Behavior. 2008;24(16):3027-44.

6. Goldberg I. Internet addiction disorder (IAD). Diagnostic criteria. Psycom.net; 1995. Disponible en: www.psycom. net/iadcriteria.html

7. Shapira NL, Goldsmith T, Szabo S, Lazoritz M, Gold M, Stein D. Problematic Internet use: proposed classification and diagnostic criteria. Depression and Anxiety. 2003;17:207-16.

8. Beard KW, Wolf EM.Modification in the proposed diagnostic criteria for internet addiction. Cyberpsychol Behav. 2001;4(3):377-83.

9. Caplan S. Problematic Internet use and psychological wellbeing: development of a theory based cognitive-behavioral measurement instrument. Computers in Human Behavior. 2002;18(5):553-75.

10. Davis RF, Besser A. Validation of a new scale for measuring problematic Internet use: implications for preemployment screening. Cyberpsychol Behav. 2002;5:331-45.

11. Charlton J. A factor-analytic investigation of computer addiction and engagement. Br J Psychol. 2002;93:329-44.

12. Charlton JD. Distinguishing addiction and high engagement in the context of online game playing. Computers in Human Behavior. 2007; 23:1531-48.

13. Ko CY, Chen C, Chen S, Yen C. Proposed Diagnostic Criteria of Internet Addiction for Adolescents. J Nerv Ment Dis. 2005;193:728-33.

14. Byun S, Ruffini C, Mills J, Douglas A, Niang M, Stepchenkova S, et al. Internet addiction: metasynthesis of 1996-2006 quantitative research. Cyberpsychol Behav. 2009;12(2):203-7.

15. Kraut PP, Lundmark V, Kiesler S, Mukopadhyay T, Scherlis W. Internet paradox: a social technology that reduces social involvement and psychological well-being? Am Psychol. 1998;53:65-77.

16. Lam LT, Peng ZW, Mai JC, Jing J. Factors associated with Internet addiction among adolescents. Cyberpsychol Behav. 2009;12(5):551-55.
17. Christakis DA. Internet addiction: a 21st century epidemic? BMC medicine. 2010;8:61.

18. Pies R. Should DSM-V Designate "Internet Addiction" a Mental Disorder? Psychiatry (Edgmont). 2009;6(2):31-7.

19. Yellowlees PM. Problematic Internet use or Internet addiction? Computers in Human Behavior. 2007;23:1447-53.

20. Peters CM. Problematic Usage Among Highly-Engaged Players of Massively Multiplayer Online Role Playing Games. Cyberpsychol Behav. 2008;11(4):481-4.

21. Ipsos APOYO Opinión y Mercado. Uso y Actitudes hacia Internet. Marketing Data [Internet]. 2008;(118):1-3.

22. Beard K. Internet Addiction: A Review of Current Assessment Techniques and Potential Assessment Questions. Cyberpsychol Behav. 2005;8(1):7-14.

23. Griffiths M. Does Internet and computer "addiction" exist? Some case study evidence. Cyberpsychol Behav. 1998;3(2):211-8

24. Huang AW, Qian M, Zhong J, Tao R. Chinese Internet Addiction Inventory: Developing a Measure of Problematic Internet Use for Chinese College Students. Cyberpsychol Behav. 2007;10(6):805-11.

25. Morahan JS. Incidence and correlates of pathological Internet use among college students. Computers in Human Behavior. 2000;16:13-29.

26. Nizama-Valladolid M. Guía para el manejo familiar de las adicciones. Modelo Familiar (afronte holístico de las adicciones). Lima: Fondo Editorial Universidad Alas Peruanas; 2001.

27. Ortiz P. Educación y formación de la personalidad. Lima: Fondo editorial-UCH; 2008.

28. Meerkerk J, Van Den Eijnden RJ, Vermulst A, Garretsen HF. The Compulsive Internet Use Scale (CIUS): some psychometric properties. Cyberpsychol Behav. 2009;12(1):1-6.

29. Nichols LA, Nicki R. Development of a psychometrically sound Internet addiction scale: a preliminary step. Psychol Addict Behav. 2004;18(4):381-4.

30. Stieger S, Burger C. Implicit and explicit self-esteem in the context of Internet addiction. Cyberpsychol Behav Soc Netw. 2010;13(6):681-8.

31. Liberatore KA, Rosario K, Martí LN, Martínez KG. Prevalence of Internet Addiction in Latino Adolescents with Psychiatric Diagnosis. Cyberpsychol Behav. Soc Netw. 2010;14(6):399-402.

Correspondencia: Nelly Lam Figueroa

Dirección: Calle Los Bananos N 195, Urb. Los Cactus, La Molina. Lima, Perú

Teléfono: 435-2726, 997-023423, 945-148453

Correo electrónico: nlamfigueroa@yahoo.es 